\title{
ADAPTIVE OPTIMAL CONTROL OF ELECTRIC POWER SYSTEM OPERATION MODE ON THE BASIS OF LEAST ACTION PRINCIPLE
}

\author{
Petro D. Lezhniuk' ', Volodymyr V. Kulyk' ${ }^{1}$, Volodymyr V. Netrebskiy' ${ }^{1}$, \\ Yevhenii V. Duchkov', Michalina Gryniewicz-Jaworska²
}

1 Department of Electrical Stations and Systems, Vinnytsia National Technical University, Vinnytsia, 21021, Ukraine

2 Lublin University of Technology, Department of Grid Network and Security, Nadbystrzycka Street 38A, 20618 Lublin, Poland, e-mail: michalina.gryniewicz.jaworska@vp.pl

Received: 2018.06 .15 Accepted: 2018.08.01 Published: 2018.09.01

\begin{abstract}
Active-adaptive system of electric power system (EPS) modes control, using its ideal ("economic") mode is suggested. The process of optimal control is constructed in such a manner that EPS current mode by means of control facilities approaches to "economic" mode. Direction, change of EPS current mode parameters are realized for its improvement and they are determined according to the least action principle (LAP). System of automatic control performs optimization actions in the direction, set by LAP, changing parameters of regulation facilities and controlls limitations of EPS mode parameters.
\end{abstract}

Keywords: electric power system, electric energy losses, mode optimization, automatic control system, least action principle.

\section{INTRODUCTION}

Electric power systems (EPS) by their nature are inhomogeneous and this characteristic feature always leads to additional losses of electric energy in the process of its transport and distribution. Availability of the existing mechanism of feedback, due to which self regulation of the system occurs, cannot provide the most profitable mode, from the point of minimal losses of electric energy and its quality. Minimizing these losses, i.e. move from natural state of EPS to optimal one, is possible only by applying forced actions. There appears the problem of necessity to change the path of EPS states in accordance with its load change. Does this require evaluating the extent to which it is possible and expedient to improve EPS mode, taking into account the limitations of mode parameters $[10,11]$.

Solution to the problem of EPS current modes approaching to optimal ones with lower losses of electric energy is realized, applying various numerical methods of linear and nonlinear programming $[1,2,7,8,9]$. Their efficiency is different but their common drawback is that they provide partial solutions, which are difficult to generalize in the laws of optimal control of EPS mode and develop corresponding system of automatic control. The paper suggests the system of adaptive optimal control of EPS normal modes using least action principle in the form of Hamilton principle.

\section{PROBLEM OF OPTIMAL COTNROL OF ELECTRIC POWER SYSTEM OPERATION MODE}

In [3] it is shown that on the basis of least action principle (LAP) system of optimal control of ESP operation modes can be built. In active-adaptive control system (AACS) of EPS normal mode, built in accordance with SMART Grid principles, where the model of its economic mode is used as 
a reference model and sources of electric energy are presented by the characteristics of economic resistances, optimal control in EPS is realized in such a way that current modes are directed and approach the ideal mode.

The problem is formulated as the problem of optimal control with limitations and fixed initial time $\mathrm{t} 0$ and fixed final time $\mathrm{tf}$, where optimality criterion is minimum losses of electric energy [4]:

$W_{\mathrm{o}}=\min \int_{\mathrm{t}_{0}}^{\mathrm{t}_{\mathrm{f}}}\left[F(\mathbf{P}, \mathbf{Q}, \mathbf{U}, \mathbf{u}, t)-F_{\mathrm{e}}\left(\mathbf{P}_{\mathrm{e}}, \mathbf{Q}_{\mathrm{e}}, \mathbf{U}_{\mathrm{e}}, \mathbf{u}_{\mathrm{e}}, t\right)\right] d t(1)$

under condition, that $\mathbf{P} \in \mathbf{D}_{\mathbf{P}}, \mathbf{Q} \in \mathbf{D}_{\mathbf{Q}}, \mathbf{U} \in \mathbf{D}_{\mathbf{U}}$, $\mathbf{u} \in \mathbf{D}_{\mathbf{u}}$,

where: $\mathrm{F}, \mathrm{F}_{\mathrm{e}}$ - functional of active power loses in current and economic (ideal from the point of electric energy losses) modes correspondingly; $\mathbf{P}, \mathbf{Q}$ and $\mathbf{P}_{\mathbf{e}}, \mathbf{Q}_{\mathbf{e}}-$ active and reactive powers of current and economic modes correspondingly; $\mathbf{U}$ and $\mathbf{U}_{\mathbf{e}}-$ voltages of EPS nodes in current and economic modes; $\mathbf{u}$ and $\mathbf{u}_{\mathrm{e}}$ - EPS control parametrs, their change from $u$ into $u e$ maximally approaches EPS mode to economic mode; $\mathbf{D}_{\mathbf{P}}, \mathbf{D}_{\mathbf{Q}}, \mathbf{D}_{\mathbf{U}}, \mathbf{D}_{\mathbf{u}}-$ admissible areas of mode $\mathbf{P}, \mathbf{Q}, \mathbf{U}$ parameters and control parameters $\mathbf{u}$ correspondingly

Figure 1 illustrates the problem (1). Approximation (optimization) of the current path of EPS mode with power losses $F_{p}$ to economic path $\mathrm{Fe}$ is realized at the expense of natural least action principle (LAP) [5] and the functioning of adaptive automatic control system (AACS) of power flows in EPS. It should be noted that in EPS as artificial engineering system, due to heterogeneity of its electric networks, the impact of

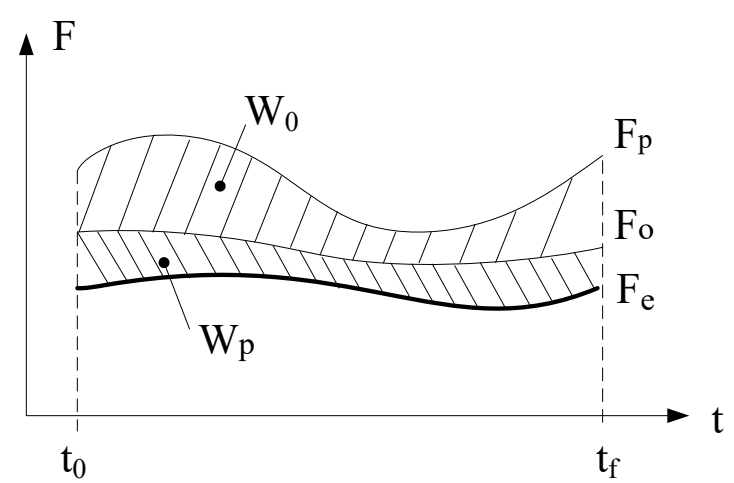

Fig. 1. Results of optimal control of EPS mode
LAP is not sufficient for the coincidence of paths $\mathrm{F}_{\mathrm{p}}$ and $\mathrm{F}_{\mathrm{e}}$. In real EPS, as a rule, due to mode parameters' limitation the coincidence of paths $F_{p}$ and $F_{e}$ cannot be achieved even by forced action of AACS. In the best case, EPS operation mode will move along the optimal path with losses $\mathrm{F}_{\mathrm{o}}$. Electric power losses $\mathrm{W}_{\mathrm{p}}$ remain uncompensated. These losses may be reduced by means of reconstruction of EPS electric networks to make them heterogeneous.

To approach (optimize) losses of electric energy to idealy possible, it is necessary to perform in the system optimizing actions by constantly regulating facilities in the process of operation. It is possible to compensate additional losses in EPS by means of voltage regulation in EPS nodes and introduction of balancing e.m.f. in the loops. In this problem control variables are e.m.f. that must be introduced in all closed loops for realization of optimal current distribution. According to economic resistances of electric power plants and sources of reactive power (SRP), their optimal loads are determined. Unlike the existing systems of optimal control, where characteristics of relative increments and simplified model of EPS are used, in AACS sources of electric energy, including renewable ones, are modelled by the characteristics of economic resistances and EPS - by its equivalent R-circuit. As information support data base of on-line information complex of EPS is used. It should be noted that in AACS, constructed in such a manner, complex optimization of EPS modes by active and reactive powers is realized [11].

\section{CALCULATION MODEL OF EPS MODES OPTIMAL CONTROL}

In [3] it is shown that the problem of EPS normal modes optimization may be reduced to calculation of economic current distribution (by equivalent R-circuit) with further introduction of the mode in admissible area. Elements of EPS, including sources of active and reactive power, are presented by active (economic) resistances $\mathrm{R}_{\mathrm{ei}}$ (see Fig. 2). For overhead transmission lines and transformers they are active components of their resistances and for the sources of energy they are determined in accordance with the problem of optimization (optimization of EPS by active, reactive power or complex optimization). 
Economic resistances of power sources $\mathrm{R}_{\mathrm{ej}}$ are calculated in accordance with the selected optimization problem, they are further specified, taking into account the obtained loads of power plants and calculated voltages in the nodes:

$$
\begin{gathered}
R_{\mathrm{ei}}=\frac{B_{\mathrm{i}}\left(P_{\mathrm{i}}\right) U_{\mathrm{i}}^{2}}{P_{\mathrm{i}}^{2}} \cdot \frac{\text { Fprice }_{\mathrm{i}}}{\text { Wcost }_{1}}, R_{\mathrm{ei}}=\frac{B_{\mathrm{j}}\left(P_{\mathrm{j}}\right) U_{\mathrm{i}}^{2}}{Q_{\mathrm{i}}^{2}} \cdot \frac{\text { Frice }_{\mathrm{j}}}{\text { Wcost }}, \\
R_{\mathrm{ei}}=\frac{B_{\mathrm{i}}\left(P_{\mathrm{i}}\right) U_{\mathrm{i}}^{2}}{\left(P_{\mathrm{i}}^{2}+Q_{\mathrm{i}}^{2}\right)} \cdot \frac{\text { Fprice }_{\mathrm{i}}}{\text { Wcost }}
\end{gathered}
$$

where: $\mathrm{B}_{\mathrm{i}}\left(\mathrm{P}_{\mathrm{i}}\right)$ - consumption characteristic of the $i^{\text {th }}$ station; $\mathrm{P}_{\mathrm{i}}$ - power of the $\mathrm{i}^{\text {th }}$ station; $\mathrm{U}_{\mathrm{i}}$ - voltage at the buses of the $\mathrm{i}^{\text {th }}$ station; $\mathrm{F}_{\text {price }}$ - price of the ton of equivalent fuel at the $\mathrm{i}^{\text {th }}$ station; $\mathrm{W}_{\text {cost }}-$ cost of 1 $\mathrm{kW} / \mathrm{h}$ of electric energy losses.

According to the first formula $\mathrm{R}_{\mathrm{ei}}$ is calculated, when EPS mode is optimized by active power, i.e., the most profitable loads of power plants are determined. According to the second formula, $\mathrm{R}_{\mathrm{ei}}$ is calculated, when EPS mode is optimized by reactive power at fixed active powers of electric stations. According to the last formula $\mathrm{R}_{\mathrm{ei}}$ is calculated, when complex optimization of EPS mode is realized. If the source of power is not a part of energy system as the entity, then instead of consumption characteristics $\mathrm{B}_{\mathrm{i}}\left(\mathrm{P}_{\mathrm{i}}\right)$ the power that is sold, is substituted and instead of $\mathrm{F}_{\text {price }}$ - price of $\mathrm{kW} / \mathrm{h} \mathrm{W}$ price $_{j}$.

Calculation model of EPS is formed and economic current distribution, which corresponds to ideal state of EPS, is determined. As in this case the second Kirchhoff's law, it is not realized in inhomogeneous networks of energy system, then contour e.m.f. of nonbalance $\dot{\mathrm{E}}_{\mathrm{nb}}$ are determined. Further, depending on the problem, two variants are possible. If steady-state mode is calculated, then the system of loop equations is formed and

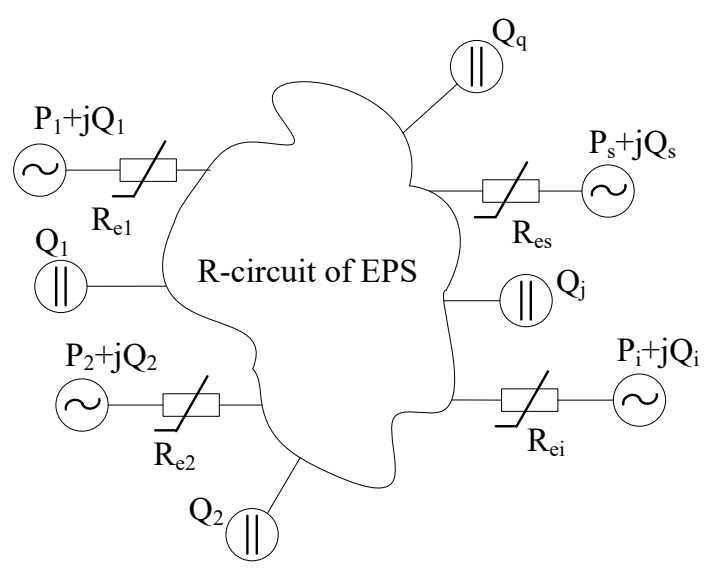

Fig. 2. Equivalent R-circuit of EPS loop currents are calculated, superposition of these currents on economic current distribution leads to realization of Kirchhoff's laws. In other variant, e.m.f. of nonbalance $\dot{\mathrm{E}}_{\mathrm{nb}}$ are calculated in transformation ratios, their introduction into EPS loops along with the change of loading of reactive power sources lead to mode balancing. This optimal mode is very close, regarding the selected optimality criterion, to ideal mode.

As transformation ratios and loading of sources of reactive power (SRP) are discrete values, then violations of limitations on voltage values in certain nodes are possible. In these cases power in generating nodes is determined and fixed that loads to realization of limitations. Power losses in ESP grow in this case.

\section{AUTOMATION OF OPTIMAL CONTROL OF EPS MODE USING LAP}

Having located the sources of electric energy by calculated resistances, we may replace the determination of total expenses minimum for generation by calculation of EPS economic mode by equivalent circuit, composed only of active resistances of EPS elements and economic resistances $\mathrm{R}_{\mathrm{ei}}$. Optimization process of electric energy sources loading can be automated in the computational environment, for instance TRACE $\operatorname{MODE}[6,12,13]$.

Adaptive control system with reference modes is well adapted for practical realization of EPS current mode approach to economic mode. The system is tested regarding EPS normal modes control, where simulation model is used as a reference model. Fig. 3 shows the adaptive system of EPS normal modes optimal control, where EPS economic mode model is used as a reference model. By means of this control system, the problem of current mode approaching to economic one, taking into account limitations is realized.

Object of control - EPS mode and regulators create the main loop of automatic control system (ACS). Disturbances in the form of load change $\mathbf{s}$ and parametric disturbance - EPS parameters deviations from their nominal value $\mathbf{q}$ constantly influence EPS mode. The value of determining parameters $\mathbf{y}^{\prime}$, their composition is determined by the results of sensitivity analysis of optimality criterion $\mathbf{F}$ to control parameters $\mathbf{u}$, are sent to the input of regulators, by means of which, according to control laws EPS mode is maintained in 
the admissible optimality area. With the help of regulators direct control impacts $\mathbf{g}$ can be realized, if necessary, by operating staff. Adaptation loop (blocks 2-5) controls regulators coefficients. Adaptation process depends, as a rule, on the vectors of external impacts $\mathbf{g}$, $\mathbf{s}$ and on parametric disturbances $\mathbf{q}$, caused by the change of meteorological conditions. Information, necessary for determination of current $\mathrm{F}$ and economic $\mathrm{F}_{\mathrm{e}}$ values of optimality criterion is sent from the database of operation information complex (OIC) into block 3 .

Block 2 is a reference model with state vector $\mathrm{xe}$, that is determined as a result of EPS steadystate mode calculation by its equivalent R-circuit. The conditions are verified in block 4 :

$$
\Delta F=\left|F-F_{\mathrm{e}}\right| \leq \xi_{\mathrm{F}}
$$

where: $\xi_{\mathrm{F}}-$ admissible deviation of optimality criterion current values from its economic value.

If the latter condition is satisfied, it means that EPS mode is in optimality area and any control actions are required. In other case, at the input of unit $5 \Delta \mathrm{F}$ is sent and changes in control laws $\mathrm{r}$, that realize change of electric energy sources generation and power from EPS and reduce $\Delta \mathrm{F}$ to $\xi_{\mathrm{F}}$ are determined.

\section{CONCLUSIONS}

The suggested approach to creation of active-adaptive control system of EPS mode, using its ideal (economic) modes enables to make

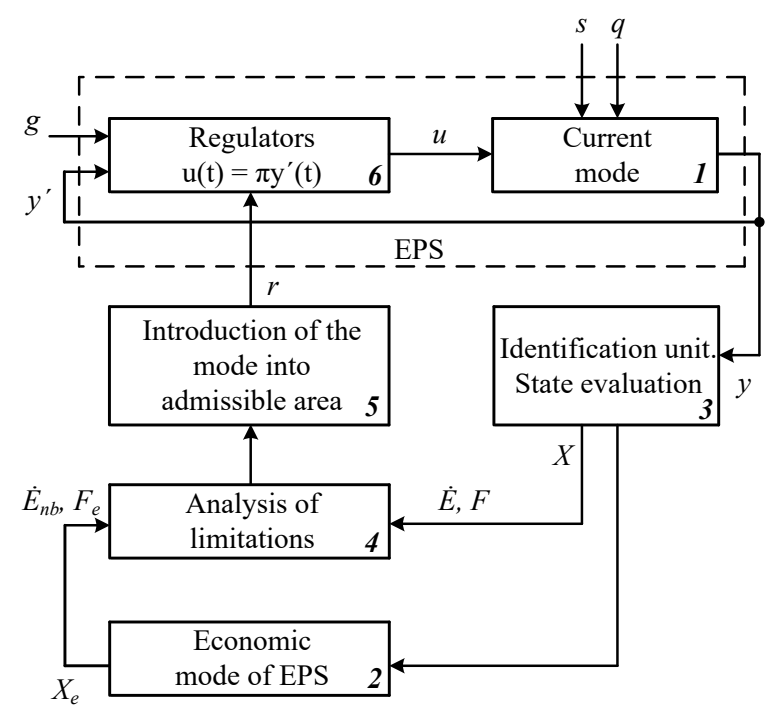

Fig. 3. Adaptive system of control with the model of EPS economic mode optimal control process more rational. It is built in such a manner that EPS mode, using control facilities, is introduced in optimality area, i.e., it approaches to economic mode as close as mode parameters limitations (voltage of the nodes, flows of power in branches, etc.) allow. The advantage of the given approach is that the system of optimal control, built in such a way, does not require the determination of numerical value of optimality criterion and corresponding parameters of EPS mode. The direction, in which changes of EPS current mode are realized for its improvement are determined by natural action according to least action principle. The task of automatic control system is reduced to maintaining of this motion, changing the parameters of regulating devices and controlling limitations on parameters of EPS mode.

\section{REFERENCES}

1. Burbelo M.J., Pijarski P., Zavadskiy V., Koczorowska-Gazda A., Lyudmyla M. Melnychuk L. M., Loboda Y. V. Measurement of reactive power under asymmetrical nonsinusoid modes of electric networks with earthed neutral. Proceedings of SPIE, 2016.

2. Feinman R., Leiton P., Sends M. Feinmanian lectures on physics Least action principle. M., MIR. Publishing House, 1966, 94-119.

3. Kacejko P., Machowski J., Pijarski P. Standing phase angle reduction at switching operation in transmission network. Rynek Energii nr 5(96), 2011, 24-35.

4. Kacejko P., Pijarski P. Connecting of wind farms limitations instead of oversized investment. Rynek Energii nr 1(80), 2009, 10-15.

5. Kacejko P., Pijarski P. Cost sharing on the network upgrade individual investors applying for connection to a network of wind farms. Przegląd Elektrotechniczny. (9a)88, 2012, 27-31.

6. Kacejko P., Pijarski P. Dynamic Fitting of Generation Level to Thermal Capacity of Overhead Lines. Przegląd Elektrotechniczny, 84(5), 2008, 80-83.

7. Kothari D.P., Dhillon J.S.. Power system optimization. Prentice-Hall of India, New Delhi 2007.

8. Kyrylenko O.V., Segeda M.S., Butkevytch O.F. Mazur T.A. Mathematical modeling in electric power engineering. Lviv: Lviv Polytechnika, Publishing House, 2013, 608.

9. Lezhniuk P.D., Kulyk V.V. Usage of least action principle as the mechanism of natural optimization in SMART GRID technologies. Tehnichna electrodynamika 4, 2014, 32-34. 
10. Montusiewicz J., Gryniewicz Jaworska M., Pijarski P.: Looking for the optimal location for wind farms. Advances in science and technology research journal. 27(9), 2015, 135-142.

11. Sage A.P., White Ch, C. Optimum systems control. Prentice-Hall, Inc. Englewood Cliffs, New Jersey, 1977.

12. Trace Mode SCADA/HMI is a high-tech soft- ware platform for process automation, remote control, monitoring, energy/resources metering and building automation. Access: http://www. tracemode.com.

13. Wydra M., Kacejko P. Power system state estimation accuracy enhancement using temperarture measurements of overhead line conductors. Metrol. Meas. Syst., 23(2), 2016, 183-192. 